\title{
Synthesis of 2-amino alcohols and unnatural amino acids from serine
}

\author{
Victoria Magrioti, Georgia Antonopoulou, Evlambia Pantoleon, and George Kokotos* \\ Laboratory of Organic Chemistry, Department of Chemistry, University of Athens, Athens \\ 15771, Greece \\ E-mail: gkokotos@cc.uoa.gr
}

\begin{abstract}
Dedicated to Professor Gerasimos J. Karabatsos on the occasion of his $70^{\text {th }}$ birthday (received 28 Feb 03; accepted 07 Jul 03; published on the web 08 Jul 03)
\end{abstract}

\begin{abstract}
An efficient route for the synthesis of enantiopure unnatural $\alpha$-amino acids and 2-amino alcohols was developed. The synthesis is based on the Wittig-type olefination of 3-benzyloxy-2-(tertbutoxycarbonylamino)propanal with various ylides.
\end{abstract}

Keywords: 2-Amino alcohols, amino aldehydes, serine, unnatural amino acids, Wittig reaction

\section{Introduction}

The synthesis of unnatural amino acids has attracted special attention in recent years. ${ }^{1,2}$ Unnatural amino acids play an important role in the design and synthesis of pharmacologically relevant molecules, peptidomimetics and enzyme inhibitors. ${ }^{3-5}$ Aldehydes obtained from natural amino acids constitute a class of chiral synthons useful in the synthesis of optically active bioactive compounds and, in particular, in the synthesis of unnatural amino acids. Aldehyde 1, known as Garner's aldehyde, ${ }^{6}$ is prepared from serine and has been used extensively in asymmetric synthesis. $N, N$-Dibenzylamino aldehydes 2 have been proven particularly useful in C-C bond forming processes. ${ }^{7}$ More recently, $N, N$-bisBoc-glutamate and aspartate aldehydes 3 have been proposed as chiral intermediates for the synthesis of unnatural amino acids ${ }^{8,9}$ and have found many applications. ${ }^{10}$

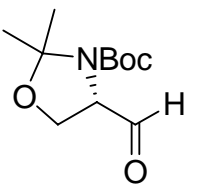

1

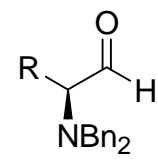

2

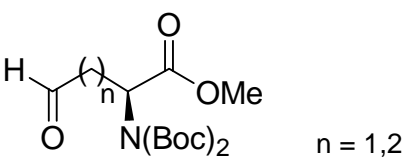

3 
Long-chain 2-amino alcohols display interesting biological activities. These activities (immunosuppresive, anti-inflammatory, cytotoxic, induction of apoptosis) together with the methods for the synthesis of long-chain 2-amino alcohols have been reviewed recently. ${ }^{11}$

This work aims at the development of an efficient method for the synthesis of unnatural amino acids and 2-amino alcohols starting from serine.

\section{Results and Discussion}

$O$-Benzyl- $N$-(tert-butoxycarbonyl)-L-serinol (4), prepared as described in literature, ${ }^{12,13}$ was oxidized to aldehyde $5^{14-16}$ (Scheme 1 ) with $\mathrm{NaOCl}$ in the presence of a catalytic amount of 4acetamido-2,2,6,6-tetramethylpiperidin-1-yloxy radical (AcNH-TEMPO). ${ }^{17,18}$ N-Protected $\alpha$ aminoaldehydes may be prepared either by reduction of a carboxy derivative of an amino acid or by oxidation of 2-aminoalcohols, and it is known that they have a high tendency for racemization. ${ }^{19}$ The $\mathrm{NaOCl} / \mathrm{TEMPO}$ method was chosen because it appears superior to reductive methods in terms of preservation of the enantiomeric purity. ${ }^{20}$

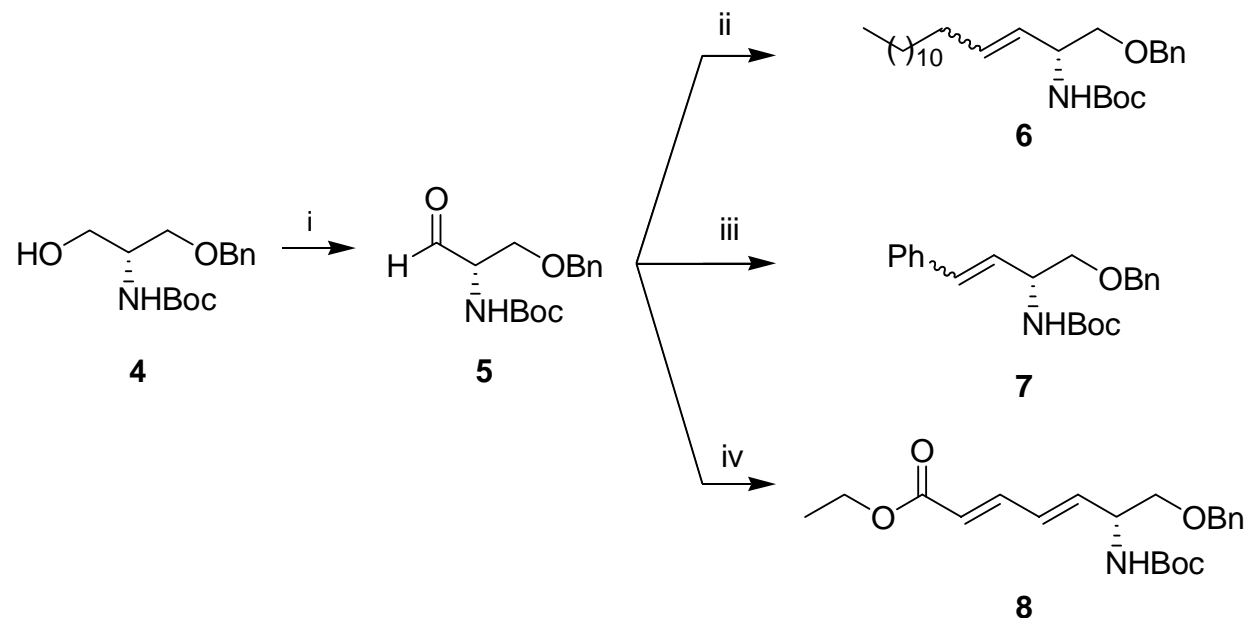

Scheme 1. Synthesis and Wittig reactions of amino aldehyde 5. (i) AcNH-TEMPO, NaOCl, $\mathrm{NaBr}, \mathrm{MePh} / \mathrm{EtOAc} / \mathrm{H}_{2} \mathrm{O}, \mathrm{NaHCO}_{3}$. (ii) $\mathrm{C}_{13} \mathrm{H}_{27} \mathrm{P}^{+}(\mathrm{Ph})_{3} \mathrm{Br}^{-}$, KHMDS. (iii) $\mathrm{PhCH}_{2} \mathrm{P}^{+}(\mathrm{Ph})_{3} \mathrm{Br}^{-}$, KHMDS, PhMe. (iv) $\mathrm{EtO}_{2} \mathrm{CCH}=\mathrm{CHCH}_{2} \mathrm{P}(=\mathrm{O})(\mathrm{OEt})_{2}, \mathrm{LiOH}$, mol. sieves, THF.

Our strategy to synthesize unnatural $\alpha$-amino acids was based on a Wittig-type olefination reaction of aldehyde $\mathbf{5}$. This useful chiral synthon should be used immediately after its preparation without any purification. Thus, compound 5 reacted with ylides that were generated by treatment of tridecyltriphenylphosphonium bromide and benzyltriphenylphosphonium bromide with potassium bis(trimethylsilyl)amide (KHMDS) in toluene at $0^{\circ} \mathrm{C}$ (Scheme 1). Under these reaction conditions, compound $\mathbf{6}$ was obtained as $Z / E$ mixture, whereas compound $\mathbf{7}$ mainly as $E$ isomer (>95\%), as shown by ${ }^{1} \mathrm{H}$ NMR spectroscopic analysis. The Horner- 
Wadsworth-Emmons olefination reaction of this aldehyde with the phosphonate anion, generated from ethyl (2E)-4-(diethoxyphosphoryl)but-2-enoate by treatment with $\mathrm{LiOH}^{21}$ afforded compound 8.

Catalytic hydrogenation of the double bonds of compounds 6-8 with simultaneous removal of benzyl group produced Boc-protected 2-amino alcohols 9-11, respectively (Scheme 2). The Bocprotected $\alpha$-amino acids 12 and $\mathbf{1 3}$ were obtained by oxidation of $\mathbf{9}$ and 10, respectively, using 2.5 equivalents of $\mathrm{NaOCl}$ in the presence of AcNH-TEMPO, and tetrabutylammonium hydrogensulfate as a phase transfer catalyst.

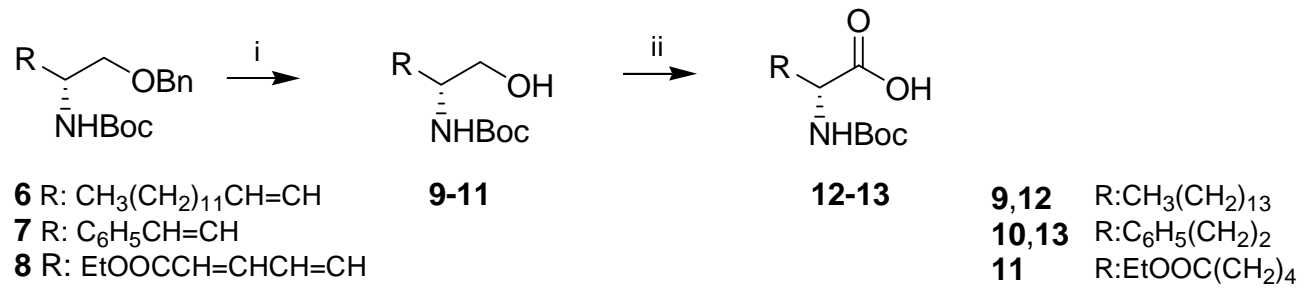

Scheme 2. Synthesis of 2-amino alcohols and unnatural amino acids. i) $\mathrm{H}_{2}, 10 \% \mathrm{Pd} / \mathrm{C}, \mathrm{MeOH}$; ii) AcNH-TEMPO, $\mathrm{NaOCl},(n-\mathrm{Bu})_{4} \mathrm{~N}^{+} \mathrm{HSO}_{4}^{-}, \mathrm{NaBr}, \mathrm{CH}_{2} \mathrm{Cl}_{2}, \mathrm{NaHCO}_{3}$.

The enantiomeric purity of the final products, 2-amino alcohols and $\alpha$-amino acids, depends on the conditions used for both the preparation of aldehyde $\mathbf{5}$ and the Wittig-type reaction. Compounds 9-11 were converted with $(S)-(-)$ and $(R)-(+)-\alpha$-methoxy- $(\alpha-$ trifluoromethyl)phenylacetic acid ${ }^{22}$ almost quantitatively into esters. ${ }^{1} \mathrm{H}$ and ${ }^{19} \mathrm{~F}$ NMR analysis of these Mosher esters indicated an enantiomeric excess $>95 \%$. The proposed method produced optically pure amino acids $\mathbf{1 2}$ and $\mathbf{1 3}$ as indicated by comparison of their specific rotation values with those reported in the literature. ${ }^{23,24}$

In conclusion, a general method for the synthesis of enantiopure unnatural $\alpha$-amino acids and 2-amino alcohols has been developed using 3-benzyloxy-2-(tert-butoxycarbonylamino)propanal 6 as the key intermediate. The strengths of the method are (1) simplicity and efficiency, and (2) flexibility with respect to the side chains that can be introduced through the olefination reaction.

\section{Experimental Section}

General Procedures. Specific rotations were measured on polarimeter using a $10 \mathrm{~cm}$ cell. NMR spectra were recorded on a $200 \mathrm{MHz}\left({ }^{1} \mathrm{H}\right.$ NMR) spectrometer. For analytical TLC plates silica gel $60 \mathrm{~F}_{254}$ and for column chromatography silica gel 60 (70-230 or 230-400 mesh) were used (Merck). Dry solvents (THF, toluene, $\mathrm{Et}_{2} \mathrm{O}$ ) were were used. $N$-Methylmorpholine was distilled from ninhydrin. All other solvents and chemicals were of reagent grade and used without further purification. The phosphonium salts were prepared by refluxing $\mathrm{PPh}_{3}$ and the corresponding alkyl halide in MeCN and were used for the Wittig reactions without purification. 
(2S)-3-Benzyloxy-2-(tert-butoxycarbonylamino)propanal (5). To a cold $\left(-5{ }^{\circ} \mathrm{C}\right)$, vigorously stirred biphasic mixture of $4^{12,13}$ (200 $\mathrm{mg}, 0.71 \mathrm{mmol}$ ) ) and 4-acetamido-TEMPO (3 mg, $0.014 \mathrm{mmol})$ in toluene $(2.1 \mathrm{~mL})$ and EtOAc $(2.1 \mathrm{~mL})$, and $\mathrm{NaBr}(80 \mathrm{mg}, 0.78 \mathrm{mmol})$ in water $(0.35 \mathrm{~mL})$ was added dropwise over a period of $2 \mathrm{~h}$ an aqueous solution $(1.84 \mathrm{~mL})$ of $\mathrm{NaOCl}$ (58.1 mg, $0.78 \mathrm{mmol})$ containing $\mathrm{NaHCO}_{3}(177 \mathrm{mg}, 2.1 \mathrm{mmol})$. The aqueous layer was separated and washed with EtOAc $(5 \mathrm{~mL})$. The combined organic phases were washed consecutively with a solution of $\mathrm{KI}(8 \mathrm{mg})$ in aqueous citric acid $(1 \%, 2 \mathrm{~mL})$, an aqueous solution of sodium thiosulfate $(10 \%, 2 \mathrm{~mL})$, and brine; after drying $\left(\mathrm{Na}_{2} \mathrm{SO}_{4}\right)$ the solvent was evaporated. The residue was immediately used in a Wittig reaction.

\section{Wittig reaction. General procedure}

To a stirred suspension of the appropriate phosphonium salt $(1.20 \mathrm{mmol})$ in dry toluene $(5.0 \mathrm{~mL})$ was added dropwise over a period of $5 \mathrm{~min}$ at $0{ }^{\circ} \mathrm{C}$ under $\mathrm{N}_{2}$ a solution of KHMDS in toluene $(0.5 \mathrm{M}, 2.4 \mathrm{~mL})$. The bright red solution was stirred for another $10 \mathrm{~min}$, and a solution of the aldehyde 5 (279 mg, $1.00 \mathrm{mmol})$ in dry toluene $(5 \mathrm{~mL})$ was added in one portion. The light yellow mixture was stirred at room temperature for $20 \mathrm{~h}$. Then, the reaction mixture was quenched with a saturated aqueous solution of $\mathrm{NH}_{4} \mathrm{Cl}(50 \mathrm{~mL})$ and extracted with $\mathrm{Et}_{2} \mathrm{O}(3 \times 30 \mathrm{~mL})$. The combined organic phases were washed with brine and dried $\left(\mathrm{Na}_{2} \mathrm{SO}_{4}\right)$. The solvent was removed, and the residue was purified by column chromatography using a mixture of EtOAc/petroleum ether as eluent.

(2R)-1-Benzyloxy-2-(tert-butoxycarbonylamino)-3-hexadecene (6). Colorless oil (263 mg, 59\%). ${ }^{1} \mathrm{H}$ NMR $\left(\mathrm{CDCl}_{3}\right): \delta 7.25-7.40(5 \mathrm{H}, \mathrm{m}, \mathrm{Ph}), 5.30-5.60(2 \mathrm{H}, \mathrm{m}, \mathrm{CH}=\mathrm{CH}), 4.82(1 \mathrm{H}, \mathrm{bs}$, $\mathrm{NH})$, 4.50-4.65 (3H, m, CHNH, $\left.\mathrm{CH}_{2} \mathrm{Ph}\right), 3.40-3.60\left(2 \mathrm{H}, \mathrm{m}, \mathrm{OCH}_{2} \mathrm{CH}\right), 2.00-2.15(2 \mathrm{H}, \mathrm{m}$, $\left.\mathrm{CH}_{2} \mathrm{CH}=\right), 1.45\left[9 \mathrm{H}, \mathrm{s}, \mathrm{C}\left(\mathrm{CH}_{3}\right)_{3}\right], 1.20-1.40\left(20 \mathrm{H}, \mathrm{m}, \mathrm{CH}_{2}\right), 0.89\left(3 \mathrm{H}, \mathrm{t}, J=6.8 \mathrm{~Hz}, \mathrm{CH}_{3}\right) .{ }^{13} \mathrm{C}$ NMR $\left(\mathrm{CDCl}_{3}\right): \delta 155.2,138.1,133.3,131.3,128.5,127.7,127.2,79.2,73.1,72.7,48.0,32.5$, 31.9, 29.6, 29.3, 28.4, 28.3, 27.8, 22.7, 14.1. Anal. Calcd. for $\mathrm{C}_{28} \mathrm{H}_{47} \mathrm{NO}_{3}$ : C, 75.46, H, 10.63, N, 3.14. Found: C, $75.38, \mathrm{H}, 10.70, \mathrm{~N}, 3.07$.

(2R, 3E)-1-Benzyloxy-2-(tert-butoxycarbonylamino)-4-phenyl-3-butadiene (7). White solid (240 mg, 68\%); mp 96-98 ${ }^{\circ} \mathrm{C} .{ }^{1} \mathrm{H}$ NMR $\left(\mathrm{CDCl}_{3}\right): \delta 7.20-7.50(10 \mathrm{H}, \mathrm{m}, 2 \mathrm{Ph}), 6.58(1 \mathrm{H}, \mathrm{d}, J=$ $16.0 \mathrm{~Hz}, \mathrm{PhCH}=\mathrm{CH}), 6.22(1 \mathrm{H}, \mathrm{dd}, J=16.0 \mathrm{~Hz}, J=6.0 \mathrm{~Hz}, \mathrm{PhCH}=\mathrm{CH}), 5.00(1 \mathrm{H}, \mathrm{bs}, \mathrm{NH})$, 4.40-4.65 (3H, m, CHNH, $\left.\mathrm{CH}_{2} \mathrm{Ph}\right), 3.61\left(2 \mathrm{H}, \mathrm{m}, \mathrm{OCH}_{2} \mathrm{CH}\right), 1.45$ [9H, s, $\left.\mathrm{C}\left(\mathrm{CH}_{3}\right)_{3}\right] .{ }^{13} \mathrm{C} \mathrm{NMR}$ $\left(\mathrm{CDCl}_{3}\right): \delta 155.3,137.9,136.7,131.3,128.4,127.8,126.2,79.5,73.2,72.3,48.0$, 28.4. Anal. Calcd. for $\mathrm{C}_{22} \mathrm{H}_{27} \mathrm{NO}_{3}$ : C, 74.76, H, 7.70, N, 3.96. Found: C, 74.85, H, 7.59, N, 3.85.

Ethyl (6R)-7-benzyloxy-6-(tert-butoxycarbonylamino)-2,4-heptadienoate (8). A suspension of aldehyde 5 (279 mg, $1.00 \mathrm{mmol}$ ), ethyl (2E)-4-(diethoxyphosphoryl)but-2-enoate (275 mg,

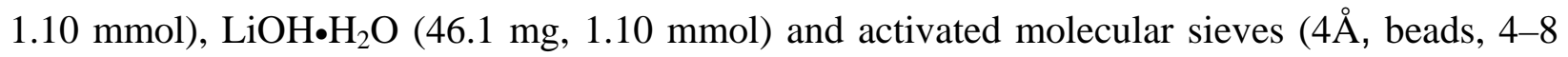
mesh, $1.5 \mathrm{~g})$ in dry THF $(10 \mathrm{~mL})$ was refluxed under a nitrogen atmosphere for $16 \mathrm{~h}$. The crude reaction mixture was filtered through Celite ${ }^{\circledR}$ eluting with ether. The solvent was evaporated under reduced pressure, and the residue was purified by column chromatography using petroleum ether/EtOAc (9:1, v/v) as eluent. Yellowish oil (177 mg, 47\%). ${ }^{1} \mathrm{H}$ NMR $\left(\mathrm{CDCl}_{3}\right): \delta$ 
7.25-7.40 (6H, m, Ph, EtOOCCH=CH), 6.00-6.40 (2H, m, EtOOCH=CHCH=CH), $5.87(1 \mathrm{H}, \mathrm{d}$, $J=15.4 \mathrm{~Hz}, \mathrm{EtOOCH}=\mathrm{CH}), 4.92(1 \mathrm{H}, \mathrm{b}, \mathrm{NH}), 4.30-4.55$ (3H, m, CHNH, $\left.\mathrm{CH}_{2} \mathrm{Ph}\right), 4.24$ (2H, q, $\left.J=7.4 \mathrm{~Hz}, \mathrm{CH}_{3} \mathrm{CH}_{2} \mathrm{O}\right), 3.55\left(2 \mathrm{H}, \mathrm{m}, \mathrm{CH}_{2} \mathrm{CHNH}\right), 1.45$ [9H, s, $\left.\mathrm{C}\left(\mathrm{CH}_{3}\right)_{3}\right], 1.25$ (3H, t, $J=7.4 \mathrm{~Hz}$, $\left.\mathrm{CH}_{3} \mathrm{CH}_{2} \mathrm{O}\right) .{ }^{13} \mathrm{C}$ NMR $\left(\mathrm{CDCl}_{3}\right): \delta 167.1,155.2,144.6,142.5,138.3,133.3,131.5,129.1,128.6$, 126.2, 79.5, 73.2, 72.3, 60.1, 48.0, 28.4, 14.2. Anal. Calcd. for $\mathrm{C}_{28} \mathrm{H}_{47} \mathrm{NO}_{3}$ : C, 67.18, $\mathrm{H}, 7.79, \mathrm{~N}$, 3.73. Found: C, 67.03, H, 7.95, N, 3.57.

\section{Catalytic hydrogenation. General procedure}

To a solution of $\mathbf{6}, 7$ or $8(1.00 \mathrm{mmol})$ in $\mathrm{MeOH}(10 \mathrm{~mL})$ was added $\mathrm{Pd} / \mathrm{C}(10 \%, 60 \mathrm{mg})$. The reaction mixture was stirred under $\mathrm{H}_{2}(1 \mathrm{~atm})$ at room temperature for $16 \mathrm{~h}$. After filtration through a pad of Celite, the solvent was removed, and the product was purified by column chromatography using petroleum ether/EtOAc (7:3, v/v) as eluent.

(2R)-2-(tert-Butoxycarbonylamino)-1-hexadecanol (9). White solid (329 mg, 92\%); mp 51$54^{\circ} \mathrm{C}$ (lit. $\left.{ }^{25} 54-55{ }^{\circ} \mathrm{C}\right) ;[\alpha]_{\mathrm{D}}=+8.5$ (c 1.0, $\left.\mathrm{CHCl}_{3}\right)$, lit. ${ }^{25}[\alpha]_{\mathrm{D}}=+8.6$ (c 1.0, $\left.\mathrm{CHCl}_{3}\right) .{ }^{1} \mathrm{H} \mathrm{NMR}$ $\left(\mathrm{CDCl}_{3}\right): \delta 4.65(1 \mathrm{H}, \mathrm{b}, \mathrm{NH}), 3.63\left(2 \mathrm{H}, \mathrm{m}, \mathrm{CH}_{2} \mathrm{OH}\right), 3.52(1 \mathrm{H}, \mathrm{m}, \mathrm{CHNH}), 2.60(1 \mathrm{H}, \mathrm{s}, \mathrm{OH})$, $1.48\left(2 \mathrm{H}, \mathrm{m}, \mathrm{CH}_{2} \mathrm{CHNH}\right), 1.44\left[9 \mathrm{H}, \mathrm{s}, \mathrm{C}\left(\mathrm{CH}_{3}\right)_{3}\right], 1.20-1.30\left(24 \mathrm{H}, \mathrm{m}, \mathrm{CH}_{2}\right), 0.87(3 \mathrm{H}, \mathrm{t}, J=$ 6.8Hz, $\left.\mathrm{CH}_{3}\right) .{ }^{13} \mathrm{C} \mathrm{NMR}\left(\mathrm{CDCl}_{3}\right): \delta 156.6,79.6,66.1,52.9,31.9,31.5,29.6,29.5,29.3,28.4$, 28.3, 28.2, 26.0, 22.7, 14.1 .

(2R)-2-(tert-Butoxycarbonylamino)-4-phenylbutanol (10). White solid (230 mg, 87\%); mp 78$80{ }^{\circ} \mathrm{C} ;[\alpha]_{\mathrm{D}}=+5.8\left(c\right.$ 1.0, $\left.\mathrm{CHCl}_{3}\right), \operatorname{lit}^{26}[\alpha]_{\mathrm{D}}=+4.5\left(\right.$ c 1.1, $\left.\mathrm{CHCl}_{3}\right) .{ }^{1} \mathrm{H} \mathrm{NMR}\left(\mathrm{CDCl}_{3}\right): \delta 7.05-$ 7.40 (5H, m, Ph), 4.66 (1H, b, NH), 3.40-3.80 (3H, m, $\left.\mathrm{CH}_{2} \mathrm{OH}, \mathrm{CHNH}\right), 2.70$ (2H, m, $\left.\mathrm{CH}_{2} \mathrm{Ph}\right)$, 2.30 (1H, b, OH), 1.60-1.85 (2H, m, $\left.\mathrm{CH}_{2} \mathrm{CHNH}\right), 1.45$ [9H, s, C $\left.\left(\mathrm{CH}_{3}\right)_{3}\right] .{ }^{13} \mathrm{C} \mathrm{NMR}\left(\mathrm{CDCl}_{3}\right): \delta$ 156.6, 141.5, 128.4, 128.3, 126.0, 79.7, 65.9, 52.6, 33.3, 32.4, 28.4.

Ethyl (6R)-7-hydroxy-6-(tert-butoxycarbonylamino)heptanoate (11). Yellowish oil (142 mg, 49\%); ${ }^{1} \mathrm{H}$ NMR $\left(\mathrm{CDCl}_{3}\right): \delta 4.75(1 \mathrm{H}, \mathrm{b}, \mathrm{NH}), 4.12$ (2H, q, $\left.J=7.2 \mathrm{~Hz}, \mathrm{CH}_{3} \mathrm{CH}_{2} \mathrm{O}\right), 3.45-3.70$ (3H, $\left.\mathrm{m}, \mathrm{CH}_{2} \mathrm{OH}, \mathrm{CHNH}\right), 2.70(1 \mathrm{H}, \mathrm{b}, \mathrm{OH}), 2.32\left(2 \mathrm{H}, \mathrm{m}, \mathrm{CH}_{2} \mathrm{COOC}_{2} \mathrm{H}_{5}\right), 1.55-1.75(2 \mathrm{H}, \mathrm{m}$, $\left.\mathrm{CH}_{2} \mathrm{CH}\right), 1.35-1.55$ [11H, m, $\left.\mathrm{CH}_{2} \mathrm{CH}_{2} \mathrm{COOC}_{2} \mathrm{H}_{5}, \mathrm{C}\left(\mathrm{CH}_{3}\right)_{3}\right], 1.20-1.35\left(5 \mathrm{H}, \mathrm{m}, \mathrm{CH}_{3} \mathrm{CH}_{2} \mathrm{O}, \mathrm{CH}_{2}\right)$.

${ }^{13} \mathrm{C} \mathrm{NMR}\left(\mathrm{CDCl}_{3}\right): \delta$ 173.6, 156.4, 79.5, 65.7, 60.3, 52.6, 34.1, 31.1 , 28.3, 25.5, 24.7, 14.2. Anal. Calcd. for $\mathrm{C}_{14} \mathrm{H}_{27} \mathrm{NO}_{5}$ : C, 58.11, H, 9.40, N, 4.84. Found: C, 58.35, H, 9.14, N, 4.77.

\section{General procedure for the oxidation of alcohols 9-11}

To a cold $\left(0{ }^{\circ} \mathrm{C}\right)$, rapidly stirred solution of $\mathbf{1 0}, 11$ or $12(1.00 \mathrm{mmol})$ in $\mathrm{CH}_{2} \mathrm{Cl}_{2}(2.5 \mathrm{~mL})$ and $\mathrm{H}_{2} \mathrm{O}$ (5 mL) were subsequently added 4-acetamido-TEMPO free radical (2 mg, $0.01 \mathrm{mmol}$ ), $\left[\mathrm{CH}_{3}\left(\mathrm{CH}_{2}\right)_{3}\right]_{4} \mathrm{~N}^{+} \mathrm{HSO}_{4}{ }^{-}(85 \mathrm{mg}, 0.25 \mathrm{mmol})$ and $\mathrm{NaBr}(10 \mathrm{mg}, 0.10 \mathrm{mmol}$,). Then a solution of $\mathrm{NaOCl}$ (186 mg, $2.50 \mathrm{mmol}$ ) in $\mathrm{H}_{2} \mathrm{O}\left(7.1 \mathrm{~mL}\right.$ ) containing $\mathrm{NaHCO}_{3}$ (355 mg) was added, and the mixture was stirred vigorously for $20 \mathrm{~min}$. The organic solvent was evaporated under reduced pressure, and the residue was taken up with EtOAc $(20 \mathrm{~mL})$ and aqueous citric acid $(10 \%, 10 \mathrm{~mL})$ containing KI (60 mg). The aqueous phase was extracted again with EtOAc $(10 \mathrm{~mL})$, and the combined organic phases were washed with aq. $\mathrm{Na}_{2} \mathrm{~S}_{2} \mathrm{O}_{3}(10 \%, 10 \mathrm{~mL})$ and brine, and dried 
$\left(\mathrm{MgSO}_{4}\right)$. The organic solvent was evaporated under reduced pressure, and the residue was purified by column chromatography using a mixture of $\mathrm{CHCl}_{3} / \mathrm{MeOH}(9: 1)$ as eluent.

(2R)-2-(tert-Butoxycarbonylamino)hexadecanoic acid (12). White solid (248 $\mathrm{mg}, 67 \%)$; $\mathrm{mp}$ 40-42 ${ }^{\circ} \mathrm{C}\left(\right.$ lit. $\left.^{23} 41^{\circ} \mathrm{C}\right) ;[\alpha]_{\mathrm{D}}=+7.7$ (c 1.0, $\left.\mathrm{CHCl}_{3}\right)\left\{\right.$ lit. $^{23}[\alpha]_{\mathrm{D}}=+7.9\left(\right.$ c 2.0, $\left.\left.\mathrm{CHCl}_{3}\right)\right\} .{ }^{1} \mathrm{H} \mathrm{NMR}$ $\left(\mathrm{CDCl}_{3}\right): \delta 5.02(1 \mathrm{H}, \mathrm{b}, \mathrm{NH}), 4.30(1 \mathrm{H}, \mathrm{m}, \mathrm{CHNH}), 1.84(1 \mathrm{H}, \mathrm{m}, \mathrm{CHHCHNH}), 1.65(1 \mathrm{H}, \mathrm{m}$, CHHCHNH), 1.45 [9H, s, C $\left.\left(\mathrm{CH}_{3}\right)_{3}\right], 1.20-1.30\left(24 \mathrm{H}, \mathrm{m}, \mathrm{CH}_{2}\right), 0.88\left(3 \mathrm{H}, \mathrm{t}, J=6.8 \mathrm{~Hz}, \mathrm{CH}_{3}\right) .{ }^{13} \mathrm{C}$ NMR $\left(\mathrm{CDCl}_{3}\right): \delta 177.6,155.6,80.0,53.4,32.4,31.8,29.8,29.7,29.6,29.5,29.4,29.3,28.3$, 25.5, 22.6, 14.0 .

(2R)-2-(tert-Butoxycarbonylamino)-4-phenylbutanoic acid (13). White oil (207 mg, 74\%); $[\alpha]_{\mathrm{D}}=+5.6(c$ 1.0, EtOH $)\left\{\right.$ lit. $^{24}[\alpha]_{\mathrm{D}}=+5.9(c$ 1.4, EtOH $\left.)\right\} .{ }^{1} \mathrm{H}$ NMR $\left(\mathrm{CDCl}_{3}\right): \delta 7.23-7.32(5 \mathrm{H}$, $\mathrm{m}, \mathrm{Ph}), 5.16(1 \mathrm{H}, \mathrm{b}, \mathrm{NH}), 4.40(1 \mathrm{H}, \mathrm{m}, \mathrm{CHNH}), 2.75\left(2 \mathrm{H}, \mathrm{m}, \mathrm{PhCH}_{2}\right), 2.05-2.22(2 \mathrm{H}, \mathrm{m}$, $\left.\mathrm{CH}_{2} \mathrm{CHNH}\right), 1.48\left[9 \mathrm{H}, \mathrm{s}, \mathrm{C}\left(\mathrm{CH}_{3}\right)_{3}\right] .{ }^{13} \mathrm{C} \mathrm{NMR}\left(\mathrm{CDCl}_{3}\right): \delta 176.6,157.0,140.6,128.4,128.3$, 126.0, 80.0, 48.0, 33.5, 31.5, 28.3.

\section{Acknowledgements}

This work was supported in part by the University of Athens (Special Account for Research Grants).

\section{References}

1. Duthaler, R.O. Tetrahedron 1994, 50, 1539.

2. Nájera, C. Synlett 2002, 9, 388.

3. Giannis, A.; Kolter, T. Angew. Chem., Int. Ed. 1993, 32, 1244.

4. Goody, R.S.; Alexandrov, K.; Engelhard, M. ChemBioChem 2002, 3, 399.

5. Wang, L.; Schultz, P.G. Chem. Commun. 2002, 1.

6. Garner, P.; Park, J.M. Org. Synth. 1992, 70, 18.

7. Reetz, M. Chem. Rev. 1999, 99, 1121.

8. Kokotos, G.; Padron, J.M.; Martin, T.; Gibbons, W.A.; Martin, V.S. J. Org. Chem. 1998, 63, 3741.

9. Markidis, T.; Kokotos, G.; J. Org. Chem. 2002, 67, 1685.

10. Constantinou-Kokotou, V.; Magrioti, V. Amino Acids 2003, 24, 231.

11. Constantinou-Kokotou, V. Lett. Peptide Sci. 2003, 9, 143.

12. Kokotos, G. Synthesis 1990, 299.

13. Kokotos, G.; Noula, C. J. Org. Chem. 1996, 61, 6994.

14. Stanfield, C.F.; Parker, J.E.; Kanellis, P. J. Org. Chem. 1981, 46, 4797.

15. Luly, J.R.; Dellaria, J.F.; Plattner, J.J.; Soderquist, J.L.; Yi, N. J. Org. Chem. 1987, 52, 1487. 
16. Sakaitani, M.; Ohfune, Y. J. Am. Chem. Soc. 1990, 112, 1150.

17. Ma, Z.; Bobbit, J. M. J. Org. Chem. 1991, 56, 6110.

18. Leanna, M.R.; Sowin, T.J.; Morton, H.E. Tetrahedron Lett. 1992, 33, 5029.

19. Jurczak, J.; Golebiowski, A. Chem. Rev. 1989, 89, 149.

20. Jurczak, J.; Gryko, D.; Kobrzycka, E.; Gruza, H.; Prokopowicz, P. Tetrahedron 1998, 54, 6051.

21. Takacs, J.M.; Jaber, M.R.; Clement, F.; Walters, C. J. Org. Chem. 1998, 63, 6757.

22. Dale, J.A.; Dull, D.L.; Mosher, H. J. Org. Chem. 1969, 34, 2543.

23. Kokotos, G.; Padron, J.M.; Noula, C.; Gibbons, W.A.; Matrin, V Tetrahedron: Asymmetry 1996, 7, 857.

24. Jackson, R.F.W.; Wishart, N.; Wood, A.; James, K.; Wythes, M.J. J. Org. Chem. 1992, 57, 3397.

25. Kokotos, G.; Constantinou-Kokotou, V.; Noula, C.; Hadjipavlou-Litina, D. Lipids 1999, 34, 307.

26. Reginato, G.; Mordini, A.; Caracciolo, M. J. Org. Chem. 1997, 62, 6187. 